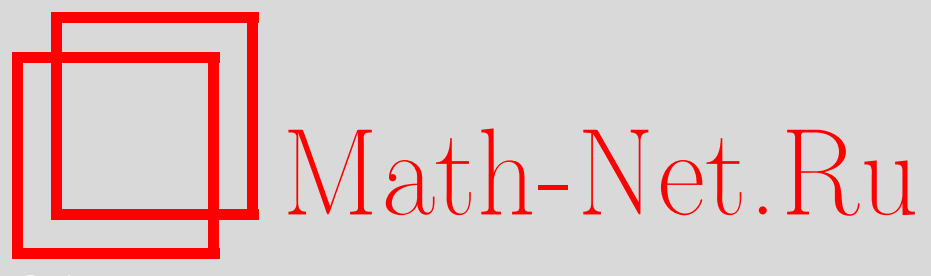

Ш. Левенталь, Х. Салехи, С. А. Чобанян, Общие максимальные неравенства, связанные с усиленным законом больших чисел, Матем. заметки, 2007, том 81, выпуск 1, 98-111

DOI: https://doi.org/10.4213/mzm3520

Использование Общероссийского математического портала Math-Net.Ru подразумевает, что вы прочитали и согласны с пользовательским соглашением http://www . mathnet.ru/rus/agreement

Параметры загрузки:

IP : 3.81 .55 .215

26 апреля 2023 г., $17: 25: 12$

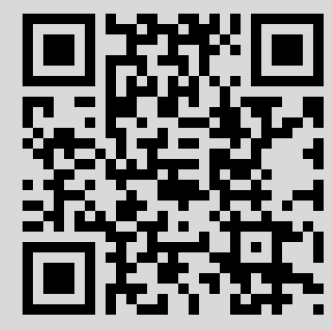


Том 81 выпуск 1 январь 2007

УДК $519.2+517.51+517.98$

\section{Общие максимальные неравенства, связанные с усиленным законом больших чисел}

\section{Ш. Левенталь, Х. Салехи, С. А. Чобанян}

Для последовательности $\left(\xi_{n}\right)$ случайных величин найдены максимальные неравенства, из которых можно вывести условия п.н. сходимости к нулю нормированных разностей

$$
\frac{1}{2^{n}}\left(\max _{2^{n} \leqslant k<2^{n+1}}\left|\sum_{i=2^{n}}^{k} \xi_{i}\right|-\left|\sum_{i=2^{n}}^{2^{n+1}-1} \xi_{i}\right|\right) .
$$

Сходимость к нулю этой последовательности приводит к усиленному закону больших чисел (УЗБЧ). В частном случае квазистационарных последовательностей мы находим достаточное условие для УЗБЧ, лучшее чем известные условия Ф. Морица.

Библиография: 14 названий.

1. Введение и обозначения. $(\Omega, \mathscr{A}, \mathrm{P})$ будет обозначать основное вероятностное пространство. Пусть $(X,\|\cdot\|)$ - банахово пространство (действительное или комплексное). Мы говорим, что $\xi: \Omega \rightarrow X-$ случайная величина со значениями в банаховом пространстве $X$ ( $X$-значная случайная величина), если она измерима по Бохнеру. $\mathbb{N}$ будет обозначать множество натуральных чисел, $\mathbb{N}_{0}$ - множество натуральных чисел и нуль, $\mathbb{R}^{n}-n$-мерное евклидово пространство, $\mathbb{C}-$ пространство комплексных чисел.

Пусть $\left(\xi_{k}\right), k \in \mathbb{N}_{0},-$ последовательность случайных величин в банаховом пространстве $X$. Обозначим

$$
\begin{aligned}
S_{b, n} & =\sum_{k=b}^{b+n-1} \xi_{k}, \quad b \in \mathbb{N}_{0}, \quad n \in \mathbb{N}, \\
M_{b, n} & =\max _{1 \leqslant k \leqslant n}\left\|S_{b, k}\right\|, \quad b \in \mathbb{N}_{0}, \quad n \in \mathbb{N} .
\end{aligned}
$$

Типичным максимальным неравенством, которое мы доказываем в этой заметке, является следующее: для любого $p, 1<p<\infty$, и любого $N \in \mathbb{N}$

$$
\sum_{n=0}^{N} \sup _{k \in \mathbb{N}_{0}} \mathrm{E}\left(\frac{M_{k, 2^{n}}^{p}-\left\|S_{k, 2^{n}}\right\|^{p}}{2^{n p}}\right) \leqslant \frac{2^{p+1}}{2^{p}-2} \sum_{n=0}^{N} G_{n, p},
$$

Работа выполнена при частичной поддержке U.S. Civilian Research and Development Foundation, Award GEMI-3328-TB-03.

(C) Ш. ЛЕвенталь, Х. САЛЕХи, С. А. ЧовАнян, 2007 
где

$$
G_{n, p}=\sup _{k \in \mathbb{N}_{0}}\left(\frac{1}{2} \mathrm{E}\left\|\frac{S_{k, 2^{n}}}{2^{n}}\right\|^{p}+\frac{1}{2} \mathrm{E}\left\|\frac{S_{k+2^{n}, 2^{n}}}{2^{n}}\right\|^{p}-\mathrm{E}\left\|\frac{S_{k, 2^{n+1}}}{2^{n+1}}\right\|^{p}\right) .
$$

Когда банахово пространство $X$ имеет специальную геометрию (например, когда оно гильбертово, конечномерно, одномерно), $G_{n, p}$ принимает весьма простой вид (см. заключительную часть этого пункта). Неравенства такого типа представляются полезными при изучении усиленного закона больших чисел (УЗБЧ).

Мы говорим, что УЗБЧ выполняется для последовательности $\left(\xi_{k}\right) \quad X$-значных случайных величин, и пишем $\left(\xi_{k}\right) \in \operatorname{SLLN}(X)$, если $^{1}$

$$
\frac{1}{n} \sum_{0}^{n-1} \xi_{k} \rightarrow 0 \quad \text { п.н. в } X .
$$

Следующее элементарное утверждение устанавливает связь между (1.1) и УЗБЧ.

ПреДЛОЖЕНИЕ 1 [1]. Пусть $\left(\xi_{k}\right), k \in \mathbb{N}_{0}$, - последовательность $X$-значных случайных величин. Тогда $\left(\xi_{k}\right) \in \operatorname{SLLN}(X)$ тогда и только тогда, когда

$$
\frac{1}{2^{n}} M_{2^{n}, 2^{n}} \rightarrow 0 \quad \text { n.н. npu } n \rightarrow \infty \text {. }
$$

Согласно предложению 1 из (1.1) вытекает, что конечность $\sum_{0}^{\infty} G_{n, p}$, вместе с п.н. сходимостью к нулю последовательности $S_{0,2^{n}} / 2^{n}$, обеспечивает УЗБЧ. Мы показываем, что в частности для гармонизуемой последовательности $\left(\xi_{n}\right)$ случайных величин (действительных или комплексных) правая часть (1.1) ограничена, так что для такой последовательности УЗБЧ эквивалентен п.н. сходимости к нулю последовательности $S_{0,2^{n}} / 2^{n}$. Этот факт впервые был установлен В. Ф. Гапошкиным сначала для стационарных последовательностей в фундаментальной работе [2], а затем им же для гармонизуемых последовательностей (и процессов) в [3]. Позже этот результат был повторен в работе Ж. Руссо-Эжель [4], а в работе К. Худре [5] он был перенесен на более широкий класс процессов. Указанная эквивалентность была главным звеном как в работах Гапошкина, так и в последующих работах при доказательстве следующего необходимого и достаточного условия для УЗБЧ для гармонизуемых процессов:

$$
Z\left(-\frac{1}{2^{n}}, \frac{1}{2^{n}}\right) \rightarrow 0 \quad \text { п.н., }
$$

где $Z$ - спектральная случайная мера процесса.

Во втором пункте мы доказываем основное рекуррентное соотношение для получения максимальных неравенств (лемму 1). В третьем пункте доказывается, что для любой ограниченной последовательности $\left(\xi_{n}\right)$ в $L_{p}(X), 1<p \leqslant 2$, существует такая последовательность $\left(b_{n}\right) \subset \mathbb{N}_{0}$, что

$$
\sum_{n=0}^{\infty} \mathrm{E} \frac{M_{b_{n}, 2^{n}}^{p}-\left\|S_{b_{n}, 2^{n}}\right\|^{p}}{2^{n p}}<\infty .
$$

\footnotetext{
${ }^{1}$ При определении УЗБЧ обычно требуют сходимость к некоторой $X$-значной случайной величине $\eta$ (не обязательно к нулю). Данное выше определение с точностью до центрирования, в настоящей статье не ограничивает общности. Для нас важно, в частности, что если $\left(\xi_{k}\right)-$ стационарная в широком смысле (или гармонизуемая) последовательность, для которой средние сходятся к $\eta$, то $\left(\xi_{k}-\eta\right)$ - также стационарная в широком смысле (соответственно, гармонизуемая) последовательность.
} 
Это соотношение обеспечивает п.н. сходимость к нулю разности

$$
\frac{M_{b_{n}, 2^{n}}-\left\|S_{b_{n}, 2^{n}}\right\|}{2^{n}}
$$

В п. 4 приводится наше основное максимальное неравенство (1.1).

В п. 5 и п. 6 мы обсуждаем приложения к УЗБЧ. В качестве следствия из неравенства (1.1) мы получаем, что УЗБЧ для последовательности $\left(\xi_{n}\right) \subset L_{p}(X)$ всегда выполняется, если для некоторого $p, 1<p<\infty$,

$$
\sum_{n=0}^{\infty} \sup _{k \in \mathbb{N}_{0}} \mathrm{E}\left\|\frac{S_{k, 2^{n}}}{2^{n}}\right\|^{p}<\infty
$$

Этот факт был известен ранее лишь для скалярных случайных величин, $p=2$ и некоторых дополнительных условиях (см. Ф. Мориц [6]). Условие (1.2) может быть проверено эффективно, например, в случае квазистационарных последовательностей. В этом случае (1.2) переходит в

$$
\sum_{m=1}^{\infty} \frac{f(m)}{m}<\infty
$$

где $f$ - квазиковариация последовательности $\left(\xi_{n}\right)$. Ранее достаточность (1.3) была известна лишь при дополнительном условии монотонности $f$. Как показал Гапошкин [2], (1.3) является в некотором смысле наилучшим достаточным условием для УЗБЧ в классе квазистационарных последовательностей.

В заключительном седьмом пункте доказывается обобщение теоремы Гапошкина, из которого, в частности, вытекает, что если $\sum_{0}^{\infty} G_{n, p}<\infty$, то

$$
\sum_{n=1}^{\infty} \mathrm{E} \max _{2^{n}<k \leqslant 2^{n+1}}\left\|\frac{S_{0, k}}{k}-\frac{S_{0,2^{n}}}{2^{n}}\right\|^{p}<\infty .
$$

Найденный в настоящей работе метод доказательства позволяет распространять "одномерные" результаты на случай банахова пространства простой заменой абсолютного значения на норму. Однако вид правой части (1.1) существенно упрощается для пространств со специальной геометрией. Например, в случае гильбертова пространства в правой части (1.1) $G_{n, p}$ можно заменить гораздо более простым и прозрачным выражением (с точностью до постоянного множителя)

$$
K_{n, p}=\sup _{k \in \mathbb{N}_{0}} \mathrm{E}\left\|\frac{S_{k, 2^{n}}-S_{k+2^{n}, 2^{n}}}{2^{n}}\right\|^{p} .
$$

Следует отметить, что взаимосвязь между $\left\|S_{b, k}\right\|$ и $M_{b, k}$ (без классических условий независимости или мартингальных условий) изучалась многими авторами. Отметим три известные работы: Морица [7], Лонгнекера-Серфлинга [8] и Серфлинга [9]. Однако наши неравенства, исследующие в общей ситуации разность между этими величинами, по-видимому, являются новыми и относятся к другому направлению. 
2. Одно общее максимальное неравенство. Для заданной последовательности $\left(\xi_{k}\right) \subset L_{p}(X), 1<p<\infty$, обозначим

$$
\begin{aligned}
& A_{n}=\sup _{k \in \mathbb{N}_{0}} \mathrm{E} M_{k, 2^{n}}^{p}, \quad n \in \mathbb{N}_{0}, \\
& B_{n}=\sup _{k \in \mathbb{N}_{0}} \mathrm{E}\left\|S_{k, 2^{n}}\right\|^{p}, \quad n \in \mathbb{N}_{0} .
\end{aligned}
$$

Лемма 1. Имеет место следующее неравенство:

$$
A_{n+1} \leqslant\left(2^{p-1}+1\right) A_{n}+\left(2^{p-1}-1\right) B_{n}, \quad n \in \mathbb{N}_{0} .
$$

ДокАЗАТЕльство. Мы имеем для любых $k \in \mathbb{N}_{0}$ и $n \in \mathbb{N}_{0}$

$$
M_{k, 2^{n+1}} \leqslant \max \left\{M_{k, 2^{n}},\left\|S_{k, 2^{n}}\right\|+M_{k+2^{n}, 2^{n}}\right\} .
$$

Используя элементарное неравенство $|a+b|^{p} \leqslant 2^{p-1}\left(|a|^{p}+|b|^{p}\right)$, получаем

$$
\begin{aligned}
M_{k, 2^{n+1}}^{p} & \leqslant \max \left\{M_{k, 2^{n}}^{p}, 2^{p-1}\left(\left\|S_{k, 2^{n}}\right\|^{p}+M_{k+2^{n}, 2^{n}}^{p}\right)\right\} \\
& \leqslant\left(2^{p-1}-1\right)\left\|S_{k, 2^{n}}\right\|^{p}+M_{k, 2^{n}}^{p}+2^{p-1} M_{k+2^{n}, 2^{n}}^{p} .
\end{aligned}
$$

Беря математические ожидания от обеих сторон (2.2), мы приходим к (2.1). Лемма доказана.

Обозначим далее

$$
D_{n}=\frac{A_{n}}{2^{p n}}, \quad E_{n}=\frac{B_{n}}{2^{p n}}, \quad n \in \mathbb{N}_{0} .
$$

Лемма 2. $\operatorname{Eсли~}\left(\xi_{k}\right) \subset L_{p}(X), 1<p<\infty$, mo

$$
\sum_{1}^{\infty}\left(D_{n}-E_{n}\right) \leqslant \frac{2^{p}}{2^{p-1}-1} A_{0}
$$

ДокАзАТЕЛЬСтво. Моментное неравенство (2.2) дает

$$
2^{p} D_{n+1} \leqslant\left(2^{p-1}+1\right) D_{n}+\left(2^{p-1}-1\right) E_{n}, \quad n \in \mathbb{N}_{0} .
$$

Суммируя это соотношение по $n$, получаем

$$
\sum_{1}^{N+1} 2^{p} D_{n} \leqslant \sum_{0}^{N}\left(\left(2^{p-1}+1\right) D_{n}+\left(2^{p-1}-1\right) E_{n}\right), \quad N \in \mathbb{N}_{0} .
$$

Отсюда

$$
2^{p} D_{N+1}+\left(2^{p-1}-1\right) \sum_{1}^{N}\left(D_{n}-E_{n}\right) \leqslant\left(2^{p-1}+1\right) D_{0}+\left(2^{p-1}-1\right) E_{0}=2^{p} A_{0} .
$$

Лемма доказана.

ЗАмечАниЕ. Леммы 1 и 2 остаются справедливыми, если $A_{n}$ и $B_{n}$ определить следующим способом:

$$
A_{n}=\sup _{l \in \mathbb{N}_{0}+m} \mathrm{E} M_{k_{0}+l \cdot 2^{n}, 2^{n}}^{p}, \quad B_{n}=\sup _{l \in \mathbb{N}_{0}+m} \mathrm{E}\left\|S_{k_{0}+l \cdot 2^{n}, 2^{n}}\right\|^{p},
$$

где $k_{0}, m \in \mathbb{N}_{0}$ фиксированы. 
3. Максимальное неравенство для ограниченной последовательности в $L_{p}(X), 1<p \leqslant 2$.

Теорема 1. Пусть $\left(\xi_{k}\right)$ - последовательность в $L_{p}(X), 1<p<\infty$. Тогда

(i) имеет место неравенство

$$
\inf _{\left(b_{n}\right)} \sum_{n=0}^{\infty} \mathrm{E} \frac{M_{b_{n}, 2^{n}}^{p}-\left\|S_{b_{n}, 2^{n}}\right\|^{p}}{2^{n p}} \leqslant \frac{2^{p}}{2^{p-1}-1} \sup _{k \in \mathbb{N}_{0}} \mathrm{E}\left\|\xi_{k}\right\|^{p}
$$

где инфимум берется по всем последовательностям $\left(b_{n}\right), b_{n} \in \mathbb{N}_{0}, n \in \mathbb{N}_{0}$;

(ii) если последовательность $\xi_{n}$ ограничена в $L_{p}(X)$, то существует такая последовательность $\left(b_{n}\right), b_{n} \in \mathbb{N}_{0}$, что

$$
\frac{1}{2^{n}}\left(M_{b_{n}, 2^{n}}-\left\|S_{b_{n}, 2^{n}}\right\|\right) \rightarrow 0 \quad \text { n.н. } и \text { в } L_{p}
$$

npu $n \rightarrow \infty$.

$B$ (i) $u$ (ii) можно ограничитъся последовательностями $b_{n}$ вида $b_{n}=l(n) \cdot 2^{n}$, где $l(n) \in \mathbb{N}_{0}$.

Заметим, что в общем случае из (3.1) не вытекает, что $M_{b_{n}, 2^{n}} / 2^{n} \rightarrow 0$ п.в.

ДоКАЗАТЕЛЬСТво теОРемЫ 1. (i) Воспользуемся леммой 2 , где $A_{n}$ и $B_{n}$ определяются формулой (2.3). По определению $B_{n}$ для любого $\varepsilon>0$ существует такая последовательность $l(n), n \in \mathbb{N}_{0}$, что

$$
B_{n}-\mathrm{E}\left\|S_{l(n) \cdot 2^{n}, 2^{n}}\right\|^{p} \leqslant \frac{2^{p}-1}{2^{p}} \varepsilon .
$$

По лемме 2

$$
\sum_{n=0}^{\infty} \mathrm{E} \frac{M_{b_{n}, 2^{n}}^{p}-\left\|S_{b_{n}, 2^{n}}\right\|^{p}}{2^{n p}} \leqslant \sum_{n=0}^{\infty}\left(D_{n}-E_{n}+\frac{2^{p}-1}{2^{p+n p}} \varepsilon\right)<\frac{2^{p}}{2^{p-1}-1} A_{0}+\varepsilon,
$$

где $b_{n}=l(n) \cdot 2^{n}$. Часть (i) доказана.

(ii) Из (i) вытекает, что для некоторой последовательности $\left(b_{n}\right)$ левая часть $(3.2)$ конечна. Поскольку для каждого $n \in \mathbb{N}_{0} M_{b_{n}, 2^{n}} \geqslant\left\|S_{b_{n}, 2^{n}}\right\|$, мы находим

$$
\frac{M_{b_{n}, 2^{n}}^{p}-\left\|S_{b_{n}, 2^{n}}\right\|^{p}}{2^{n p}} \rightarrow 0 \quad \text { п.н., }
$$

что доказывает (ii).

4. Основное максимальное неравенство. Из максимального неравенства, доказываемого в теореме 2 этого пункта, можно получить, в частности, условия п.н. сходимости к нулю разности $\left(M_{k, 2^{n}}-\left\|S_{k, 2^{n}}\right\|\right) / 2^{n}$. Нам представляется, что оно может иметь и самостоятельный интерес.

Пусть $X-$ банахово пространство, $1 \leqslant p<\infty$. Для заданной последовательности $\left(\xi_{k}\right) \subset L_{p}(X)$ обозначим

$$
\begin{aligned}
F_{n, p} & =\sup _{k \in \mathbb{N}_{0}} \mathrm{E}\left(\frac{M_{k, 2^{n}}^{p}-\left\|S_{k, 2^{n}}\right\|^{p}}{2^{n p}}\right), \\
G_{n, p} & =\sup _{k \in \mathbb{N}_{0}}\left(\frac{1}{2} \mathrm{E}\left\|\frac{S_{k, 2^{n}}}{2^{n}}\right\|^{p}+\frac{1}{2} \mathrm{E}\left\|\frac{S_{k+2^{n}, 2^{n}}}{2^{n}}\right\|^{p}-\mathrm{E}\left\|\frac{S_{k, 2^{n+1}}}{2^{n+1}}\right\|^{p}\right), \\
K_{n, p} & =\sup _{k \in \mathbb{N}_{0}} \mathrm{E}\left\|\frac{S_{k, 2^{n}}-S_{k+2^{n}, 2^{n}}}{2^{n}}\right\|^{p} .
\end{aligned}
$$


Tеорема 2. Пусть $X$ - банахово пространство, $1<p<\infty, \alpha \geqslant 1$.

(i) Для любой последовательности $\left(\xi_{n}\right) \subset L_{p}(X)$ и любого $N \in \mathbb{N}$ имеем

$$
\sum_{n=0}^{N} F_{n, p}^{\alpha} \leqslant\left(\frac{2^{p+1}}{2^{p}-2}\right)^{\alpha} \sum_{n=0}^{N} G_{n, p}^{\alpha}
$$

в частности,

$$
\sum_{n=0}^{\infty}\left[\mathrm{E}\left(\frac{M_{2^{n}, 2^{n}}^{p}-\left\|S_{2^{n}, 2^{n}}\right\|^{p}}{2^{n p}}\right)\right]^{\alpha} \leqslant\left(\frac{2^{p+1}}{2^{p}-2}\right)^{\alpha} \sum_{n=0}^{\infty} G_{n, p}^{\alpha} .
$$

(ii) Если $X$ - гильбертово пространство (в частности, $X=\mathbb{R}^{1}$ или $X=\mathbb{C}$ ) и $1<p \leqslant 2$, то для любого $N \in \mathbb{N}$

$$
\sum_{n=0}^{N} F_{n, p}^{\alpha} \leqslant\left(\frac{2}{2^{p}-2}\right)^{\alpha} \sum_{n=0}^{N} K_{n, p}^{\alpha} .
$$

Доказательство. (i) Согласно (2.2) мы имеем для любого $n \in \mathbb{N}_{0}$ и $k \in \mathbb{N}_{0}$

$$
\begin{aligned}
M_{k, 2^{n+1}}^{p}-\left\|S_{k, 2^{n+1}}\right\|^{p} \leqslant & M_{k, 2^{n}}^{p}-\left\|S_{k, 2^{n}}\right\|^{p}+2^{p-1}\left(M_{k+2^{n}, 2^{n}}^{p}-\left\|S_{k+2^{n}, 2^{n}}\right\|^{p}\right) \\
& -\left\|S_{k, 2^{n+1}}\right\|^{p}+2^{p-1}\left\|S_{k, 2^{n}}\right\|^{p}+2^{p-1}\left\|S_{k+2^{n}, 2^{n}}\right\|^{p} .
\end{aligned}
$$

Разделим обе части неравенства на $2^{(n+1) p}$, возьмем ожидания от обеих сторон, а затем максимумы по всем $k$. Тогда получим

$$
F_{n+1, p} \leqslant \frac{1}{2^{p}} F_{n, p}+\frac{1}{2} F_{n, p}+G_{n, p}, \quad n \in \mathbb{N}_{0} .
$$

Индукцией по $n$ легко убедиться, что

$$
F_{n+1, p} \leqslant \sum_{k=0}^{n} c^{n-k} G_{k, p}, \quad n \in \mathbb{N}_{0},
$$

где $c=1 / 2+1 / 2^{p}$. Полагая $\alpha \geqslant 1$ и используя неравенство Йенсена, получаем

$$
F_{n+1}^{\alpha} \leqslant C(p, \alpha) \sum_{k=0}^{n} c^{n-k} G_{k}^{\alpha},
$$

где $C(p, \alpha)=\left(\sum_{0}^{\infty} c^{k}\right)^{\alpha-1}$. Суммируя (4.4) от $n=0$ до $n=N$, мы приходим к (4.1).

(ii) Известно, что если $a$ и $b$ - элементы гильбертова пространства и $1 \leqslant p \leqslant 2$, то выполняется следующее неравенство параллелограмма:

$$
\frac{\|a\|^{p}}{2}+\frac{\|b\|^{p}}{2} \leqslant\left\|\frac{a+b}{2}\right\|^{p}+\left\|\frac{a-b}{2}\right\|^{p} .
$$

Замечая, что

находим

$$
\frac{S_{k, 2^{n+1}}}{2^{n+1}}=\frac{1}{2} \frac{S_{k, 2^{n}}}{2^{n}}+\frac{1}{2} \frac{S_{k+2^{n}, 2^{n}}}{2^{n}},
$$

$$
G_{n, p}=\sup _{k \in \mathbb{N}_{0}} \mathrm{E}\left\|\frac{S_{k, 2^{n}}-S_{k+2^{n}, 2^{n}}}{2 \cdot 2^{n}}\right\|^{p}=\frac{1}{2^{p}} \sup _{k \in \mathbb{N}_{0}} \mathrm{E} \frac{\left\|S_{k, 2^{n}}-S_{k+2^{n}, 2^{n}}\right\|^{p}}{2^{n p}},
$$

что доказывает (ii). 
ЗАмЕчАниЕ 1. В действительности мы доказали большее - именно, что (ii) выполняется для некоторого $p, 1<p \leqslant 2$, когда $X$ - банахово пространство типа $p$ с константой типа, равной единице.

ЗАмечАниЕ 2. Выбирая в начальном неравенстве (4.3) доказательства $k=l \cdot 2^{n+1}$ и беря супремум по всем $l \in \mathbb{N}$, мы получаем справедливость теоремы 2 для $F_{n, p}, G_{n, p}$ и $K_{n, p}$, определенных следующим образом:

$$
\begin{aligned}
F_{n, p} & =\sup _{l \in \mathbb{N}} \mathrm{E}\left(\frac{M_{l \cdot 2^{n}, 2^{n}}^{p}-\left\|S_{l \cdot 2^{n}, 2^{n}}\right\|^{p}}{2^{n p}}\right), \\
G_{n, p} & =\sup _{l \in \mathbb{N}}\left(\frac{1}{2} \mathrm{E}\left\|\frac{S_{l \cdot 2^{n}, 2^{n}}}{2^{n}}\right\|^{p}+\frac{1}{2} \mathrm{E}\left\|\frac{S_{(l+1) \cdot 2^{n}, 2^{n}}}{2^{n}}\right\|^{p}-\mathrm{E}\left\|\frac{S_{l \cdot 2^{n}, 2^{n+1}}}{2^{n+1}}\right\|^{p}\right), \\
K_{n, p} & =\sup _{l \in \mathbb{N}} \mathrm{E}\left\|\frac{S_{2 l \cdot 2^{n}, 2^{n}-S_{(2 l+1) \cdot 2^{n}, 2^{n}}}}{2^{n}}\right\|^{p} .
\end{aligned}
$$

ЗАмЕчаниЕ 3. Существуют примеры неограниченных последовательностей $\left(\xi_{n}\right)$ в $L_{p}$, для которых правая часть в (4.1) и (4.2) ограничена (конечна).

5. Приложение к УЗБЧ. В этом пункте мы применяем теорему 2(ii) для нахождения класса последовательностей $\left(\xi_{n}\right)$, для которых УЗБЧ эквивалентен п.н. сходимости последовательности $S_{0,2^{n}} / 2^{n}$. Эта эквивалентность является главным звеном в доказательстве УЗБЧ Гапошкина [2] для стационарных в широком смысле процессов, а также в ряде последующих статей [4], [10], [5], посвященных УЗБЧ для гармонизуемых и более общих процессов, допускающих спектральное представление. Во всех этих работах метод доказательства существенно использует спектральное представление случайной последовательности (процесса). Наш метод, основанный на теореме 2, позволяет распространить результат на более широкий класс случайных последовательностей, не обязательно допускающих спектральное представление.

СлеДСТвИЕ. Пусть $X$ - банахово пространство (действительное или комплексное $),\left(\xi_{n}\right)$ - некоторая последовательность в $L_{p}(X), 1<p<\infty$. При выполнении каждого из следующих условий УЗБЧ для $\left(\xi_{n}\right)$ эквивалентен п.н. сходимости $к$ нулю последовательности $S_{0,2^{n}} / 2^{n}$ :

(i) $X$ произвольно $u \sum_{n=1}^{\infty} G_{n, p}<\infty$;

(ii) $X$ - гильбертово пространство, $1<p \leqslant 2 u \sum_{n=1}^{\infty} K_{n, p}<\infty$;

(iii) $X=\mathbb{R}^{1}$ или $X=\mathbb{C}, p=2 u \xi_{n}=A \eta_{n}, n \in \mathbb{N}_{0}$, где $\left(\eta_{n}\right)$ - стационарная в иироком смысле последовательность в $L_{2}(X)$, а $A: L_{2}(X) \rightarrow L_{2}(X)$ - линейный непрерывный оператор.

ДокАзАтеЛьство. Пусть (i) выполняется и $S_{0,2^{n}} / 2^{n} \rightarrow 0$ п.в. Легко видеть, что последнее соотношение эквивалентно сходимости $S_{2^{n}, 2^{n}} / 2^{n} \rightarrow 0$ п.н. Согласно теореме 2(i)

$$
\frac{M_{2^{n}, 2^{n}}^{p}-\left\|S_{0,2^{n}}\right\|^{p}}{2^{n p}} \rightarrow 0 \quad \text { п.н. }
$$

Отсюда вытекает п.н. сходимость к нулю последовательности $M_{2^{n}, 2^{n}} / 2^{n}$. Теперь УЗБЧ следует из предложения 1 . Эквивалентность УЗБЧ п.н. сходимости $S_{0,2^{n}} / 2^{n}$ $\rightarrow 0$ при условии (ii) доказывается аналогично. 
Пусть теперь выполнено (iii). Для того, чтобы свести доказательство этого пункта к предыдущему, нужно показать, что

$$
\sum_{n=1}^{\infty} K_{n, p}<\infty
$$

Последнее есть следствие следующей леммы.

Лемма 3. (i) Пусть $\left(\eta_{k}\right)$ - стачионарная в широком смысле последовательность (действительная или комплексная). Тогда

$$
\sum_{n} \mathrm{E}\left|\frac{S_{0,2^{n}}}{2^{n}}-\frac{S_{0,2^{n}+1}-S_{0,2^{n}}}{2^{n}}\right|^{2}<\infty .
$$

(ii) Пусть $\xi_{k}=A \eta_{k}, k \in \mathbb{N}_{0}$, где $\left(\eta_{k}\right)$ - стационарная в широком смысле последовательность, а $A: L_{2} \rightarrow L_{p}, p>1$, - линейный непрерывный оператор. Тогда

$$
\sum_{n} \sup _{k \in \mathbb{N}_{0}}\left(\mathrm{E}\left|\frac{S_{k, 2^{n}}}{2^{n}}-\frac{S_{k+2^{n}, 2^{n}}}{2^{n}}\right|^{p}\right)^{2 / p}<\infty,
$$

где $S_{k, n}=\sum_{i=k}^{k+n-1} \xi_{i}$. В частности, если $A$ действует в $L_{2}$, mо

$$
\sum_{n} \sup _{k \in \mathbb{N}_{0}} \mathrm{E}\left|\frac{S_{k, 2^{n}}}{2^{n}}-\frac{S_{k+2^{n}, 2^{n}}}{2^{n}}\right|^{2}<\infty .
$$

ДоказАтеЛьство. (i) Положим $\alpha_{n}=S_{0,2^{n}} / 2^{n}$ и $\beta_{n}=\left(S_{0,2^{n+1}}-S_{0,2^{n}}\right) / 2^{n}$. Замечая, что

$$
\alpha_{n+1}=\frac{1}{2}\left(\alpha_{n}+\beta_{n}\right)
$$

используя "равенство параллелограмма"

$$
\frac{1}{4}\left|\alpha_{n}-\beta_{n}\right|^{2}+\frac{1}{4}\left|\alpha_{n}+\beta_{n}\right|^{2}=\frac{1}{2}\left|\alpha_{n}\right|^{2}+\frac{1}{2}\left|\beta_{n}\right|^{2},
$$

стационарность последовательности $\left(\xi_{n}\right)$ и $(5.1)$, получаем

$$
\frac{1}{4} \mathrm{E}\left|\alpha_{n}-\beta_{n}\right|^{2}=\mathrm{E}\left|\alpha_{n}\right|^{2}-\mathrm{E}\left|\alpha_{n+1}\right|^{2} .
$$

Отсюда вытекает

$$
\sum_{n=0}^{\infty} \mathrm{E}\left|\alpha_{n}-\beta_{n}\right|^{2}=4 \mathrm{E}\left|\alpha_{0}\right|^{2}-\lim \mathrm{E}\left|\alpha_{n+1}\right|^{2},
$$

что доказывает часть (i). Часть (ii) леммы есть простое следствие (i). Лемма доказана.

ЗАмЕчАниЕ. Как показано в [11], каждая гармонизуемая последовательность есть линейный непрерывный образ стационарной в широком смысле последовательности. Более того, там было доказано, что каждая гармонизуемая последовательность может быть представлена как ортогональная проекция стационарной в широком смысле последовательности. Следовательно, условие (iii) эквивалентно гармонизуемости $\left(\xi_{n}\right)$. 
6. Общие моментные условия для УЗБЧ. В качестве еще одного следствия теоремы 2 мы формулируем одно моментное достаточное условие для УЗБЧ, которое улучшает и обобщает условие Морица. При $X=\mathbb{R}^{1}, p=2$ и некоторых дополнительных условиях теорема 3 была доказана в работе Морица [6].

Теорема 3. Пусть $1<p<\infty$. Если для последовательности $\left(\xi_{n}\right), n \in \mathbb{N}_{0} \subset$ $L_{p}(X)$,

$$
\sum_{n=0}^{\infty} \sup _{k \in \mathbb{N}_{0}} \mathrm{E}\left\|\frac{S_{k, 2^{n}}}{2^{n}}\right\|^{p}<\infty,
$$

то для $\left(\xi_{n}\right)$ выполняется УЗБЧ.

ДокАзАТЕЛЬСтво. Согласно (6.1) и определению $G_{n, p}$ (см. п. 4)

$$
\sum_{n=0}^{\infty} G_{n, p} \leqslant \sum_{n=0}^{\infty} \sup _{k \in \mathbb{N}_{0}} \mathrm{E}\left\|\frac{S_{k, 2^{n}}}{2^{n}}\right\|^{p}<\infty .
$$

Следовательно, по теореме 2(i)

$$
\frac{M_{2^{n}, 2^{n}}^{p}-\left\|S_{2^{n}, 2^{n}}\right\|^{p}}{2^{n p}} \rightarrow 0 .
$$

Условие (6.1) также влечет сходимость

$$
\frac{\left\|S_{2^{n}, 2^{n}}\right\|^{p}}{2^{n p}} \rightarrow 0 \quad \text { п.н. }
$$

Теперь (6.2) дает

$$
\frac{M_{2^{n}, 2^{n}}^{p}}{2^{n p}} \rightarrow 0 \quad \text { п.н., }
$$

что согласно предложению 1 эквивалентно УЗБЧ.

СлеДСТВИЕ 1. Пусть $\left(\xi_{n}\right), n \in \mathbb{N}_{0} \subset L_{p}(X)$, и для некоторого $p, 1<p<\infty, u$ всех $k, n \in \mathbb{N}_{0}$ выполняется неравенство

$$
\mathrm{E}\left\|S_{k, n}\right\|^{p} \leqslant g(n),
$$

где $g$ - некоторая неотрицательная функция на $\mathbb{N}_{0}$. Тогда

(i) если

$$
\sum_{n=1}^{\infty} \frac{g\left(2^{n}\right)}{2^{n p}}<\infty
$$

то для $\left(\xi_{n}\right)$ выполняется УЗБЧ;

(ii) если выражение $g(n) / n^{p+1}$ монотонно по $n u$

$$
\sum_{n=1}^{\infty} \frac{g(n)}{n^{p+1}}<\infty
$$

то для $\left(\xi_{n}\right)$ выполняется УЗБЧ.

ДокАЗАтЕЛЬСтво. (i) есть простое следствие теоремы 3. (ii) вытекает из (i) согласно принципу конденсации Коши. 
ЗАмечание. Часть (ii) следствия 1 в случае $X=\mathbb{R}^{1}, p=2$ представляет собой старый результат И. Гала и Дж. Коксмы [12] (см. также работу Гапошкина [2]).

Применим теорему 3 к квазистационарным последовательностям случайных величин. Пусть $f(n), n \in \mathbb{N}_{0}$, - неотрицательная функция. Мы говорим, что последовательность случайных величин (действительных или комплексных) $\left(\xi_{n}\right), n \in \mathbb{N}_{0}$, является $f$-квазистационарной, если $\mathrm{E}\left|\xi_{k}\right|^{2}<\infty, k \in \mathbb{N}_{0}$, и

$$
\left|\mathrm{E} \xi_{l} \bar{\xi}_{l+m}\right| \leqslant f(m) \quad \forall l, m \in \mathbb{N}_{0} .
$$

СлЕДСТВие 2. Пусть $\left(\xi_{n}\right), n \in \mathbb{N}_{0},-f$-квазистационарная последовательность случайных величин. Если

$$
f(0)+\sum_{m=1}^{\infty} \frac{f(m)}{m}<\infty,
$$

то для $\left(\xi_{n}\right)$ выполняется УЗБЧ.

ДокАзАтЕЛЬСтво. Для $f$-квазистационарной последовательности $\left(\xi_{n}\right), n \in \mathbb{N}_{0}$, имеем для любых $k, n \in \mathbb{N}_{0}$

$$
\mathrm{E}\left|\frac{S_{k, 2^{n}}}{2^{n}}\right|^{2} \leqslant \sum_{m=0}^{2^{n}-1} \frac{f(m)\left(2^{n}-m\right)}{2^{2 n}} \leqslant \frac{1}{2^{n}} \sum_{m=0}^{2^{n}-1} f(m) .
$$

Отсюда вытекает

$$
\begin{aligned}
\sum_{n=0}^{\infty} \sup _{k} \mathrm{E}\left|\frac{S_{k, 2^{n}}}{2^{n}}\right|^{2} & \leqslant \sum_{n=0}^{\infty} \sum_{m=0}^{2^{n}} \frac{f(m)}{2^{n}} \leqslant 2 f(0)+\sum_{m=1}^{\infty} f(m) \sum_{n=\left[\log _{2} m\right]}^{\infty} \frac{1}{2^{n}} \\
& \leqslant 2 f(0)+2 \sum_{m=1}^{\infty} \frac{f(m)}{m}
\end{aligned}
$$

Следствие 2 доказано.

Следствие 2 ранее было известно при дополнительном условии монотонности $f$. Впервые оно было установлено П. Эрдешем [13] для монотонных $f(m)=O\left(\log ^{-\alpha} m\right)$, $m \in \mathbb{N}_{0}, \alpha>1$. Гал и Коксма [12] распространили этот результат на монотонные последовательности $f(m)$, удовлетворяющие (6.3). Гапошкин [2] показал, что условие (6.3) для монотонных $f$ в некотором смысле является и необходимым: если

$$
\sum_{m=1}^{\infty} \frac{f(m)}{m}=\infty
$$

то существует $f$-квазистационарная последовательность $\left(\xi_{n}\right), n \in \mathbb{N}_{0}$, для которой УЗБЧ не выполняется.

Стоит отметить, что для стационарных в широком смысле последовательностей условие (6.3) можно заменить на более слабое условие сходимости (условной) следующего ряда:

$$
\sum_{m=1}^{\infty} \frac{R(m)}{m \log m} \log \log m
$$

где $R$ - корреляционная функция последовательности [2].

Результаты этого пункта аннонсированы в [14]. 
7. Обобщение теоремы Гапошкина. При доказательстве эквивалентности УЗБЧ и п.н. сходимости последовательности $S_{0,2^{n}} / 2^{n}$ для стационарных в широком смысле процессов Гапошкин [2], [3] (так же, как и впоследствии Руссо-Эжель [4] и Худре [5] для более широких классов процессов) в действительности доказал конечность следующего выражения:

$$
\sum_{n=0}^{\infty} \mathrm{E} \max _{2^{n}<k \leqslant 2^{n+1}}\left|\frac{S_{0, k}}{k}-\frac{S_{0,2^{n}}}{2^{n}}\right|^{2}
$$

Целью настоящего пункта является получение неравенства, обеспечивающего конечность (7.1) для класса случайных последовательностей, включающего, в частности, гармонизуемые последовательности.

Как мы отмечали в замечании 2 к теореме 2, в теореме 2 можно значительно сузить область тех $k$, по которым берется супремум (доказательство, по существу, не меняется) и сформулировать ее следующим образом (для простоты мы формулируем теорему для случая гильбертова пространства).

Теорема $2^{\prime}$. Пусть $\left(\xi_{k}\right) \subset L_{p}(X)$, где $X$ - гилъбертово пространство, $1<$ $p \leqslant 2, \alpha \geqslant 1$. Тогда для любого $N \in \mathbb{N}$ выполняется следующее неравенство:

$$
\sum_{n=0}^{N}\left[\sup _{k \in \mathbb{N}} \mathrm{E} \frac{M_{k \cdot 2^{n}, 2^{n}}^{p}-\left\|S_{k \cdot 2^{n}, 2^{n}}\right\|^{p}}{2^{n p}}\right]^{\alpha} \leqslant \frac{2}{2^{p}-2} \sum_{n=0}^{N}\left[\sup _{k \in \mathbb{N}} \mathrm{E}\left\|\frac{S_{2 k \cdot 2^{n}, 2^{n}}-S_{(2 k+1) \cdot 2^{n}, 2^{n}}}{2^{n}}\right\|^{p}\right]^{\alpha} .
$$

В этом пункте, опираясь на теорему $2^{\prime}$, мы устанавливаем следующее максимальное неравенство.

Теорема 4. Пусть $\left(\xi_{k}\right) \subset L_{p}(X)$, где $X$ - гилъбертово пространство, $1<p \leqslant 2$, $\alpha \geqslant 1$. Тогда для каждого $N \in \mathbb{N}$ выполняется следующее неравенство:

$$
\sum_{n=0}^{N}\left[\mathrm{E} \max _{2^{n}<k \leqslant 2^{n+1}}\left\|\frac{S_{0, k}}{k}-\frac{S_{0,2^{n}}}{2^{n}}\right\|^{p}\right]^{\alpha} \leqslant C(p, \alpha) \sum_{n=0}^{N}\left[\sup _{k \in \mathbb{N}_{0}} \mathrm{E}\left\|\frac{S_{2 k \cdot 2^{n}, 2^{n}}-S_{(2 k+1) \cdot 2^{n}, 2^{n}}}{2^{n}}\right\|^{p}\right]^{\alpha},
$$

где $C(p, \alpha)$ - некоторая постоянная, зависящая только от $p$ и $\alpha$.

ДоКАЗАТЕЛЬСтво. Для простоты изложения мы приводим доказательство для $\alpha=1$. Положим

$$
\eta_{0}=0, \quad \eta_{k}=\xi_{k}-\frac{S_{2^{n}, 2^{n}}}{2^{n}}, \quad 2^{n} \leqslant k<2^{n+1}, \quad n \in \mathbb{N}_{0}
$$

Легко видеть, что

$$
S_{2^{n}, 2^{n}}^{\eta}=0 \quad \text { и } \quad S_{(2 k) \cdot 2^{n}, 2^{n}}^{\eta}-S_{(2 k+1) \cdot 2^{n}, 2^{n}}^{\eta}=S_{(2 k) \cdot 2^{n}, 2^{n}}-S_{(2 k+1) \cdot 2^{n}, 2^{n}} .
$$

(Обозначение $S$ без верхнего индекса соответствует последовательности $\left(\xi_{n}\right)$.) Применим теперь теорему $2^{\prime}$ к последовательности $\left(\eta_{k}\right)$. Тогда из (7.2) и $(7.4)$ получаем

$$
\sum_{n=0}^{N} \mathrm{E} \frac{M_{2^{n}, 2^{n}}^{p}(\eta)}{2^{n p}} \leqslant C_{p} \sum_{n=0}^{N} \sup _{k \in \mathbb{N}} \mathrm{E}\left\|\frac{S_{2 k \cdot 2^{n}, 2^{n}}-S_{(2 k+1) \cdot 2^{n}, 2^{n}}}{2^{n}}\right\|^{p} .
$$


Отсюда вытекает, что

$$
\sum_{n=0}^{N} \mathrm{E} \max _{2^{n}<k \leqslant 2^{n+1}}\left\|\frac{S_{0, k}^{\eta}-S_{0,2^{n}}^{\eta}}{k}\right\| \leqslant C_{p} \sum_{n=0}^{N} \sup _{k \in \mathbb{N}} \mathrm{E}\left\|\frac{S_{2 k \cdot 2^{n}, 2^{n}}-S_{(2 k+1) \cdot 2^{n}, 2^{n}}}{2^{n}}\right\|^{p} .
$$

(Постоянная $C_{p}$ меняется от строчки к строчке.) Далее имеем

$$
\frac{S_{0, k}^{\eta}-S_{0,2^{n}}^{\eta}}{k}=\frac{S_{0, k}-S_{0,2^{n}}-\left(k-2^{n}\right) \cdot \frac{S_{2^{n}, 2^{n}}}{2^{n}}}{k}=\frac{S_{0, k}}{k}-\left[p_{k} \frac{S_{0,2^{n}}}{2^{n}}+\left(1-p_{k}\right) \frac{S_{0,2^{n+1}}}{2^{n+1}}\right],
$$

где $p_{k}=\left(2^{n+1}-k\right) / k, 2^{n} \leqslant k<2^{n+1}$. Далее,

$$
\begin{aligned}
\max _{2^{n}<k \leqslant 2^{n+1}}\left\|\frac{S_{0, k}}{k}-\frac{S_{0,2^{n}}}{2^{n}}\right\|^{p} & \\
\leqslant & 2^{p-1} \cdot \max _{2^{n}<k \leqslant 2^{n+1}}\left\{\left\|\frac{S_{0, k}}{k}-\left[p_{k} \cdot \frac{S_{0,2^{n}}}{2^{n}}+\left(1-p_{k}\right) \cdot \frac{S_{0,2^{n+1}}}{2^{n+1}}\right]\right\|^{p}\right\} \\
& +2^{p-1} \cdot \max _{2^{n}<k \leqslant 2^{n+1}}\left\{\left\|\left[p_{k} \cdot \frac{S_{0,2^{n}}}{2^{n}}+\left(1-p_{k}\right) \cdot \frac{S_{0,2^{n+1}}}{2^{n+1}}\right]-\frac{S_{0,2^{n}}}{2^{n}}\right\|^{p}\right\} \\
\leqslant & 2^{p-1} \cdot \max _{2^{n}<k \leqslant 2^{n+1}}\left\{\left\|\frac{S_{0, k}}{k}-\left[p_{k} \cdot \frac{S_{0,2^{n}}}{2^{n}}+\left(1-p_{k}\right) \cdot \frac{S_{0,2^{n+1}}}{2^{n+1}}\right] \frac{S_{0,2^{n}}}{2^{n}}\right\|^{p}\right\} \\
& +2^{p-1} \cdot\left\|\frac{S_{0,2^{n+1}}}{2^{n+1}}-\frac{S_{0,2^{n}}}{2^{n}}\right\|^{p} \cdot
\end{aligned}
$$

Простыми преобразованиями получаем

$$
\frac{S_{0,2^{n}}}{2^{n}}-\frac{S_{0,2^{n+1}}}{2^{n+1}}=\frac{1}{2}\left(\frac{S_{0,2^{n}}}{2^{n}}-\frac{S_{2^{n}, 2^{n}}}{2^{n}}\right) .
$$

Последовательно используя (7.8), (7.7), (7.6) и (7.5), находим

$$
\begin{aligned}
\sum_{n=0}^{N} \mathrm{E} & \max _{2^{n}<k \leqslant 2^{n+1}}\left\|\frac{S_{0, k}}{k}-\frac{S_{0,2^{n}}}{2^{n}}\right\|^{p} \\
\leqslant & 2^{p-1} \sum_{n=0}^{N} \max _{2^{n}<k \leqslant 2^{n+1}} \mathrm{E}\left\{\left\|\frac{S_{0, k}}{k}-\left[p_{k} \cdot \frac{S_{0,2^{n}}}{2^{n}}+\left(1-p_{k}\right) \frac{S_{0,2^{n+1}}}{2^{n+1}}\right]\right\|^{p}\right\} \\
& +\frac{2^{p-1}}{2^{p}} \sum_{n=0}^{N} \mathrm{E}\left\|\frac{S_{0,2^{n}}}{2^{n}}-\frac{S_{2^{n}, 2^{n}}}{2^{n}}\right\|^{p} \\
\leqslant & 2^{p-1} \sum_{n=0}^{N} \mathrm{E} \max _{2^{n}<k \leqslant 2^{n+1}}\left\|\frac{S_{0, k}^{\eta}-S_{0,2^{n}}^{\eta}}{k}\right\|^{p}+\frac{1}{2} \sum_{n=0}^{N} \mathrm{E}\left\|\frac{S_{0,2^{n}}}{2^{n}}-\frac{S_{2^{n}, 2^{n}}}{2^{n}}\right\|^{p} \\
\leqslant & C_{p} \sum_{n=0}^{N} \sup _{k \in \mathbb{N}_{0}} \mathrm{E}\left\|\frac{S_{2 k \cdot 2^{n}, 2^{n}}-S_{(2 k+1) \cdot 2^{n}, 2^{n}}}{2^{n}}\right\|^{p} \cdot
\end{aligned}
$$

Заметим, что супремум по $k \in \mathbb{N}_{0}$ появился только в последней строчке. Теорема доказана. 
Следующий пример показывает, что ряд в правой части (7.3) может расходиться, в то время как ряд в правой части (7.2) сходится. В этом примере левая часть (7.3) расходится, в то время как левая часть (7.2) тривиально сходится.

ПримеР. Положим $\xi_{0}=0$ и если $2^{n} \leqslant l<2^{n+1}, n \in \mathbb{N}_{0}$, то

$$
\xi_{l}= \begin{cases}1, & n \text { нечетно, } \\ 0, & n \text { четно }\end{cases}
$$

Очевидно, что для каждого $n \in \mathbb{N}_{0}$ и $k \in \mathbb{N}$

$$
S_{2 k \cdot 2^{n}, 2^{n}}-S_{(2 k+1) \cdot 2^{n}, 2^{n}}=0 .
$$

Это значит, что ряд в правой части (7.2) (тривиально) сходится, в то время как тот же ряд в правой части (7.3), с дополнительно включенной точкой $k=0$, расходится, поскольку для каждого четного $n$

$$
\frac{S_{0,2^{n}}-S_{2^{n}, 2^{n}}}{2^{n}} \geqslant \frac{1}{2}
$$

СлЕДСтвиЕ [2], [4], [5]. Если $\left(\xi_{n}\right)$ - гармонизуемая последовательность, то

$$
\sum_{n=0}^{\infty} \mathrm{E} \max _{2^{n}<k \leqslant 2^{n+1}}\left|\frac{S_{0, k}}{k}-\frac{S_{0,2^{n}}}{2^{n}}\right|^{2}<\infty .
$$

ДокАЗАТЕЛЬСтво вытекает из теоремы 4 и леммы 3, так как согласно последней ряд в правой части (7.3) сходится.

Стоит также выделить следующее более сильное утверждение, которое мы, по существу, доказали в процессе доказательства теоремы 4.

Теорема $4^{\prime}$. Пусть $\left(\xi_{k}\right) \subset L_{p}(X)$, где $X$ - гилъбертово пространство, $1<p \leqslant$ $2, \alpha \geqslant 1$. Тогда для каждого $N \in \mathbb{N}$ выполняется следующее неравенство:

$$
\begin{gathered}
\sum_{n=0}^{N}\left[\mathrm{E} \max _{2^{n}<k \leqslant 2^{n+1}}\left\|\frac{S_{0, k}}{k}-\left(p_{k} \frac{S_{0,2^{n}}}{2^{n}}+\left(1-p_{k}\right) \frac{S_{0,2^{n+1}}}{2^{n+1}}\right)\right\|^{p}\right]^{\alpha} \\
\leqslant C(p, \alpha) \sum_{n=0}^{N}\left[\sup _{k \in \mathbb{N}_{0}} \mathrm{E}\left\|\frac{S_{2 k \cdot 2^{n}, 2^{n}-S_{(2 k+1) \cdot 2^{n}, 2^{n}}}}{2^{n}}\right\|^{p}\right]^{\alpha},
\end{gathered}
$$

где $C(p, \alpha)$ - постоянная, зависящая только от $p u \alpha, p_{k}=\left(2^{n+1}-k\right) / k, 2^{n}<$ $k \leqslant 2^{n+1}, n \in \mathbb{N}_{0}$.

\section{СПИСОК ЦИТИРОВАННОЙ ЛИТЕРАТУРЫ}

[1] S. A. Chobanyan, S. Levental, V. Mandrekar, "Prokhorov blocks and strong law of large numbers under rearrangements", J. Theoret. Probab., 17:3 (2004), 647-672.

[2] В.Ф. Гапошкин, "Критерии для усиленного закона больших чисел для некоторых стационарных процессов второго порядка и однородных случайных полей", Теория вероятн. и ее применения, 22:2 (1977), 295-319. 
[3] В.Ф. Гапошкин, “Теорема о сходимости почти всюду последовательности измеримых функций и ее применения к последовательности стохастических интегралов", Maтем. сб., 104 (146):1 (1977), 3-21.

[4] J. Rousseau-Egele, "La loi forte des grands nombres pour les processus harmonisables", Ann. Inst. H. Poincaré Sect. B (N.S.), 15:2 (1979), 175-186.

[5] C. Houdré, "On the spectral SLLN and pointwise ergodic theorem in $L^{\alpha ", ~ A n n . ~ P r o b a b ., ~}$ 20:4 (1992), 1731-1753.

[6] F. Móricz, "The strong law of large numbers for quasi-stationary sequences", $Z$. Wahrscheinlichkeitstheorie und Verw. Gebiete, 38:3 (1977), 223-236.

[7] F. Móricz, "Moment inequalities and the strong laws of large numbers", Z. Wahrscheinlichkeitstheorie und Verw. Gebiete, 35:4 (1976), 299-314.

[8] M. Longnecker, R. J. Serfling, "General moment and probability inequalities for the maximum partial sum", Acta Math. Acad. Sci. Hungar., 30:1-2 (1977), 129-133.

[9] R. J. Serfling, "On the strong law of large numbers and related results for quasistationary sequences", Теория вероятн. и ее прим., 25:1 (1980), 190-194.

[10] D. Dehay, "Strong law of large numbers for weakly harmonizable processes", Stochastic Process. Appl., 24:2 (1987), 259-267.

[11] A. G. Miamee, H. Salehi, "Harmonizability, $V$-boundedness and stationarity dilation of stochastic processes", Indiana Univ. Math. J., 27:1 (1978), 37-50.

[12] I.S. Gál, J. F. Koksma, "Sur l'ordre de grandeur des fonctions sommables", Indagationes Math., 12 (1950), 192-207.

[13] P. Erdős, "On the strong law of large numbers", Trans. Amer. Math. Soc., 67:1 (1949), $51-56$.

[14] S. Chobanyan, S. Levental, H. Salehi, "Strong law of large numbers under a general moment condition", Electron. Comm. Probab., 10 (2005), 218-222.

Ш. Левенталь (S. Levental)

Поступило

Michigan State University

04.09.2004

E-mail: levental@stt.msu.edu

Исправленный вариант

Х. Салехи (Н. Salehi)

08.08.2006

Michigan State University

E-mail: salehi@msu.edu

C. А. Чобанян

Институт вычислительной математики им. Н. И. Мусхелишвили АН Грузии

E-mail: chob@gw.acnet.ge 